\title{
Realizing the Potential for HIV Self-Testing
}

\author{
C. Johnson $\cdot$ R. Baggaley $\cdot$ S. Forsythe $\cdot$ \\ H. van Rooyen - N. Ford - S. Napierala Mavedzenge • \\ E. Corbett $\cdot$ P. Natarajan $\cdot$ M. Taegtmeyer
}

Published online: 2 July 2014

(C) Springer Science+Business Media New York 2014
Keywords HIV · HIV self-testing · HIV testing and counseling $\cdot$ Testing gap $\cdot$ Rapid diagnostic test . Prevention

\section{Introduction}

HIV self-testing (HIVST), a process in which an individual performs a HIV rapid diagnostic test (RDT) and interprets the result in private [1], was first proposed in the mid-1980s [2]. At that time, due to the lack of accurate RDTs and available antiretroviral drugs, there were concerns about whether HIVST results would be reliable and how testers would respond to receiving results without professional support [3]. In recent years, given the revised World Health Organization (WHO) recommendation for earlier treatment [4], evidence that effective treatment prevents onward transmission of HIV [5], and enduring low levels of testing coverage in most settings, policy makers are turning to new testing approaches to facilitate greater uptake, earlier diagnosis, and greater access to prevention, care and treatment services. HIVST is an emerging approach that is well accepted [6], potentially costeffective [7] and empowering for those who may not otherwise test, particularly health workers, couples, men, key populations and others at high risk of HIV infection [1, 8-10].

To further explore the potential of HIVST, the Liverpool School of Tropical Medicine (LSTM) and WHO held the first global symposium on the legal, ethical, gender, human rights and public health implications of HIVST in April 2013 [11]. The meeting highlighted the potential of HIVST to increase access to and uptake of HIV testing, particularly for populations at highest risk of infection, and emphasized the need to further develop evidence around the quality of HIVST, linkage to post-test services, and the risks and the benefits associated with scale-up of HIVST [11]. This 
special issue of AIDS and Behavior links directly to the symposium and presents some of the latest research and thinking on the scale-up of HIVST.

\section{Who can Benefit from HIVST?}

Two papers in this issue provide evidence that suggests HIVST may be particularly acceptable in high burden settings. In Malawi, a qualitative study of couples HIVST within a larger cluster-randomized trial, by Kumwenda et al. [12], reports high acceptability of HIVST and indicates that HIVST can motivate partner testing and disclosure. This study reports that different forms of "persuasion" did take place and female respondents said that, due to their culture or social position, dealing with a serodiscordant HIVST result was challenging [12]. In Kenya, a cross-sectional survey and pilot implementation program by Kalibala et al. [13] found that HIVST, including couples HIVST, was acceptable to health workers. These results are in line with several surveys that report health workers in sub-Saharan Africa have been self-testing for HIV "informally" since at least 2005 [1316]. Although questions as to the appropriate programmatic approach remain, couples and health workers in high burden settings could particularly benefit from HIVST scale-up.

The results of these studies support a growing body of evidence that suggests a broad range of populations in a variety of settings are interested in or already self-testing for HIV, including serodiscordant couples, general populations [17-19], health workers [13, 15, 16], female sex workers (FSW) [20], young people [21, 22], and men who have sex with men (MSM) [23-27]. Additionally, key populations and populations at high ongoing risk for HIV who may require regular re-testing, such as serodiscordant couples or those using pre-exposure prophylaxis (PrEP) [11], may also benefit significantly from HIVST.

\section{What is the State of Policy and Regulatory Systems for HIVST?}

A review of HIVST policies by Wong et al. [28], in this issue, reports that few countries have national policies regarding self-testing, but many are considering developing or adapting policies. Those that do have policies regarding HIVST include the USA, Kenya, France, and the UK [28]. Currently the United States Food and Drug Administration (FDA) approved OraQuick ${ }^{\circledR}$ In-Home HIV Test is the only HIVST kit that meets international standards $[1,19]$. Other less rigorously evaluated diagnostics, however, are available over-the-counter or through the Internet in settings with less robust regulatory systems [1,29].
The absence of appropriate policies and regulations for medical devices, as suggested by this review, could leave HIVST and HIV RDTs unregulated with potentially deleterious implications [28]. Ensuring that retailers and service providers in resource-limited settings have the necessary policy and regulatory frameworks to support quality HIVST, while also protecting consumers from substandard products, may not be simple and will likely vary by context. Another recent legal review across seven sub-Saharan Africa countries identified numerous areas of legal uncertainty regarding HIV policies, as well as differences in how private and public sector regulations may apply to HIVST [30]. However, some key laws and policies that may need to be developed, adapted or strengthened include: the right to health, the right to privacy, liability, quality assurance, licensing of medicines or medical devices, criminalization of HIV transmission, age of consent, who can perform an HIV test and interpret the result, and where an HIV test can be distributed or performed [30]. The process of adapting, developing and strengthening policies and regulations may have challenges, but is important to guide the scale-up of HIVST.

\section{Is There an Ideal Self-Test?}

Questions remain as to what comprises an ideal HIVST kit that is accurate, easy to use, easy to interpret, and preferable to users. In this issue, Peck et al. report on a mixed methods usability study of unsupervised HIVST in South Africa, Kenya and Malawi intended to identify critical components of HIVST kits [31]. This study found that user errors were very common and suggests available HIV RDTs evaluated in this study are not ideal for HIVST. Key challenges identified with the evaluated test kits included: the lack of integrated test components, poor labeling and unclear instructions on how to perform the test and how to interpret results. Even though a majority of users made mistakes, they still felt confident when self-testing and stated they would use HIVST kits in the future [31]. This apparent disconnect between high user self-confidence and a high frequency of mistakes requires further examination. The results from this study highlight the importance of clear instructions and test system design to create an ideal HIVST kit and the need to explore different approaches to delivering instructions, such as group demonstrations and new media.

While current HIVST options may be limited, efforts to develop an ideal kit are underway and some reports are promising. According to a study of unsupervised HIVST in South Africa, after many iterations, a user-friendly finger stick kit was developed and users across rural and urban settings with minimal education were able to use the test, receive a valid and accurate result, and correctly interpret 
results [32]. With efforts to engage manufacturers, develop a full target product profile, design quality HIVST kits with optimized instructions for use, more ideal self-tests can be developed and brought to market. However, even after these tests enter the market, it will be crucial to continue evaluating their performance to make further improvements.

\section{Is HIVST Ethical?}

Ethical views on HIVST remain divergent. Two opposing perspectives on ethics are presented in this issue by Scott [33] and Allais and Venter [34]. Though opposing on the ultimate ethical consequences, both papers recognize the potential of HIVST to extend services to populations who may not otherwise test, as well as its potential to increase users' autonomy and responsibility. In particular, Allais and Venter argue HIVST should be a legally available, as it is an ethical and safe strategy to scale-up HIV testing, and has results that are arguably no more life-changing than those of other diseases and conditions, such as diabetes and pregnancy, for which self-testing is readily available and less rigorously evaluated. Thus, provided suitable regulatory and policy frameworks, quality test kits and quality assurance systems are in place, the risks are likely to be minimal and outweighed by the benefit of earlier and more equitable access to treatment [34].

In contrast, Scott [33] argues unsupervised HIVST introduces ethical dilemmas by scaling-up testing where treatment is unavailable, increasing user autonomy without support, and increasing potential risk for coercive testing, inter-partner violence, and psycho-social distress, especially in settings with pre-existing violence and among key populations who fear accessing HIV services. Moreover, HIVST will also impact legal policies that criminalize the transmission of HIV and could alter who incurs responsibility for partner-notification and linkage to HIV services [33]. Although these concerns are common for all other HIV testing approaches and not unique to HIVST, by de-coupling HIV testing from the health system, the overall responsibility of the health system may decrease; individuals may be "abandoned" without direct access to confirmatory testing and support services [33]. However, these concerns are largely unsubstantiated by evidence; as reported by Brown et al.'s review [35], harms that result from self-testing across a range of uses, including HIVST, have rarely been reported.

\section{What are the Market and Knowledge Gaps in HIVST Scale-Up?}

Estimates suggest the potential market for HIVST is vast. In South Africa alone, the Society for Family Health estimated the HIVST market is at least 2.8 million consumers annually [36]. Despite the market potential, barriers to uptake are perpetuated by the current high cost of validated HIVST kits, lack of competition within the market, limited information on demand in resource-limited settings and the need for suitable packaging and instructions for HIVST in various contexts. To achieve HIVST scale-up, strategies to reduce prices, create demand, and build on existing knowledge regarding acceptability, feasibility and implementation are needed. Through these actions interest can be stimulated among donors and manufacturers to further facilitate market entry. Otherwise, high costs will continue to create uncertainty about the future availability of affordable test kits, limit implementation science projects, and ultimately slow innovation and HIVST scale-up [37].

To address barriers to HIVST scale-up, it is important to learn from current experiences and focus on minimizing existing knowledge gaps. As reflected in this issue by Cambiano et al. [37], mathematical modeling is important to evaluate HIVST at a national and global level and understand how to best ensure HIVST has a significant impact on increasing the equity of, access to and uptake of HIV testing and counseling (HTC). In terms of current experiences, a recent cost-effectiveness analysis in $\mathrm{Zim}$ babwe showed that if HIVST cost USD3 per test, health services could save USD53 million over 20 years and have a significant public health impact [7]. A mathematical model showed that 4,000 new HIV infections could be averted in the first year of introducing the FDA approved HIVST kit in the US market [19], and another mathematical model reported that high risk MSM in the USA who have low condom use may benefit from HIVST [38]. However, a model of HIV transmission between MSM in the USA showed the potential for HIVST to increase HIV incidence on an assumption of a switch away from frequent facility-based testing using antigen-antibody combination assays and nucleic acid amplification tests to HIVST using a less sensitive oral fluid-based RDT [39]. Although this model only evaluated HIVST among individuals accessing highly accurate facility-based testing, it underscores the importance of reducing user errors through the provision of quality HIVST kits and clear instructions for use, highlighted in this issue. While evidence is promising, many factors need to be considered and included in future models and implementation science projects to fully support HIVST scale-up.

Thus, key knowledge gaps that need to be addressed include: (1) the influence HIVST may have on health seeking behaviors, (2) how different service delivery models can influence the public health impact and costeffectiveness of HIVST, (3) how the feasibility and benefits of HIVST vary by context, (4) how to best evaluate if there is any risk of substituting other HTC strategies with 
HIVST, (5) how the consequences may vary in different settings, (6) the rate of people with a positive HIVST result who receive confirmatory testing, are diagnosed HIV positive and link to treatment and care; and the rate of people who are HIV negative that link to prevention services [37]. Further, the quality of existing HTC services is suboptimal in many resource-limited and other settings [40]. Thus HIVST error rates, test accuracy, and depth of understanding key HIVST health messages should be assessed against those obtained in real health service settings that provide HTC services.

\section{How do We Realize the Potential of HIVST?}

Reaching people who do not know their HIV serostatus is an urgent global priority. Although HIVST alone will not eliminate the testing gap, it has a unique potential to reach high-risk populations who may not otherwise test. Research in this special issue finds HIVST to be acceptable and even preferable for many users. Although some stakeholders continue to have concerns about potential risks related to HIVST [10, 35], many of the vulnerabilities raised in this issue are similar to all HIV testing approaches and risks can be minimized if HIVST is provided: with clear information, with quality products, in a regulated way, within a human rights framework and with community involvement [1]. However, despite scientific progress and policy shifts, public demand for HIVST continues to outpace the public health response.

To fully maximize the opportunity HIVST presents, the public health community can support the current momentum by ensuring that: post-market surveillance systems are developed, estimates of the market size and cost-effectiveness of HIVST are enhanced, policy and regulatory systems to ensure the quality of available HIVST kits are developed, systems to monitor and report social harms, and track and identify how people who self-test are linked to prevention, care and treatment services are developed, and innovative methods and technologies are leveraged, such as mHealth and eHealth interventions. This response could result in a significant public health impact for years to come and address the needs of the evolving HIV epidemic and people living with or at risk of HIV infection worldwide.

Acknowledgments The editorial team would like to thank Dr. Elizabeth Marum for technical input and Kathryn Roberts for administrative support. This special issue was provided with support from the Brocher Foundation and the Bill and Melinda Gates Foundation.

Conflict of interest The editorial team declares no conflict of interest.

\section{References}

1. World Health Organization (WHO). March 2014 supplement to the consolidated guidelines on the use of antiretroviral drugs for treating and preventing HIV infection, recommendations for a public health approach. Geneva: WHO; 2014. p. 12-7.

2. Home HIV test kits offer privacy and convenience. AIDS Alert. 1997 [cited 2014 May 19];11(7, suppl. 1-2). http://www.ncbi. nlm.nih.gov/pubmed/11363555.

3. Pant Pai N, Klein MB. Are we ready for home-based, self-testing for HIV? Future HIV Therapy. 2008 [cited 2014 March 17]; 2(6):515-20. http://www.medscape.com/viewarticle/585706.

4. World Health Organization (WHO). Consolidated guidelines on the use of antiretroviral drugs for treating and preventing HIV infection: recommendations for a public health approach. Geneva: WHO; 2013 [cited 2014 March 17]. http://www.who.int/hiv/ pub/guidelines/arv2013/en/.

5. Cohen M, Chen ZQ, McCauleyet M, et al. Prevention of HIV-1 infection with early antiretroviral therapy. $\mathrm{N}$ Engl $\mathrm{J}$ Med. 2011;365:493-505.

6. Suthar A, Ford N, Bachanas PJ, et al. Towards universal voluntary HIV testing and counselling: a systematic review and metaanalysis of community-based approaches. PloS Med. 2013;10(8):e1001496.

7. Cambiano V, Ford D, Mabugu T, et al. Assessment of the potential cost-effectiveness of HIV self-testing in resource limited settings. In:Presented at 21st Conference for Retrovirus and Infections; Boston, MA, USA; 3-6 Mar 2014.

8. Pant Pai N, Sharma J, Shivkumar S, et al. Supervised and unsupervised self-testing for HIV in high- and low-risk populations: a systematic review. PLoS Med. 2013;10(4):e1001414.

9. Krause J, Subklew-Sehume F, Kenyon C, Colebunders R. Acceptability of HIV self-testing: a systematic literature review. BMC Public Health. 2013 [cited 2014 May 19];13(735). http:// www.biomedcentral.com/1471-2458/13/735.

10. Napierala Mavedzenge S, Baggaley R, Corbett EL. A review of self-testing for HIV: research and policy priorities in a new era of HIV prevention. Clin Infect Dis. 2013;57(1):126-38.

11. World Health Organization (WHO). Report on the first international symposium on self-testing for HIV: the legal, ethical, gender, human rights and public health implications of self-testing scale-up. Geneva: WHO; 2013. http://www.who.int/hiv/pub/ vct/self_test/en/.

12. Kumwenda M, Munthali A, Theobald S, et al. Factors shaping initial decision-making to self-test amongst cohabiting couples in urban Blantyre, Malawi. AIDS Behav. 2014. doi:10.1007/ s10461-014-0817-9.

13. Kalibala S, Tun, W, Cherutich P, Nganga A, Oweya E, Oluochet P. Factors associated with acceptability of HIV self-testing among health care workers in Kenya. AIDS Behav. 2014. doi:10. 1007/s10461-014-0830-z.

14. National AIDS and STD Control Programme (NASCOP). Preparedness for HIV/AIDS service delivery: the 2005 Kenya health worker survey. Nairobi: NASCOP; 2006 [cited 2014 March 17]. http://www.popcouncil.org/pdfs/horizons/KenyaHealthWorker Survey.pdf.

15. Corbett E. Health worker access to HIV/TB prevention, treatment and care services in Africa: situational analysis and mapping of routine and current best practices. Geneva: London School of Hygiene and Tropical Medicine, World Health Organization, Global Health Workforce Alliance; 2007.

16. Kebede B, Abate T, Mekonnen D. HIV self-testing practices among health care workers: feasibility and options for accelerating HIV testing services in Ethiopia. Pan Afr Med J. 2013 [cited 2014 
May 19];15(50). http://www.ncbi.nlm.nih.gov/pmc/articles/ PMC3786151/.

17. Choko A, MacPherson P, Webb EL, et al. One year outcomes following community-based HIV self-testing: a prospective study in Malawi. In:Presented at 21st conference of retroviruses and opportunistic infections; Boston, MA, USA; 3-6 Mar 2014

18. Choko A, MacPherson P, Webb EL, et al. The uptake and accuracy of oral kits for HIV self-testing in high HIV prevalence setting: a cross-sectional feasibility study in Blantyre, Malawi. PLoS Med. 2011;8(10):e1001102.

19. US Food and Drug Administration (FDA). OraQuick ${ }^{\circledR}$ In-Home HIV test summary of safety and effectiveness. Silver Spring: FDA; 2012 [cited 19 May 2014]. http://www.fda.gov/downloads/ BiologicsBloodVaccines/BloodBloodProducts/ApprovedProducts /PremarketApprovalsPMAs/UCM312534.pdf.

20. Napierala Mavedzenge S. personal communication, Feb 282014.

21. van Dyk A. Client-initiated, provider-initiated, or self-testing for HIV: what do South Africans prefer? J Assoc Nurses AIDS Care. 2013;24(6):e45-56.

22. Pant Pai N, Bhargava M, Joseph L, et al. Will HIV self testing be accepted by low to medium risk educated populations? a pilot cross sectional study in students of McGill University. AIDS Res Treat. 2014 [cited 2014 May 19];2014. doi:10.1155/2014/ 747619.

23. Carballo-Diéguez A, Frasca T, Balan I, Ibitoye M, Dolezal C. Use of a rapid HIV home test prevents HIV exposure in a high risk sample of men who have sex with men. AIDS Behav. 2012;16(7):1753-60.

24. Bavitinos B, Brown G, Hurley M, et al. Which gay men would increase their frequency of HIV testing with home self-testing? AIDS Behav. 2013;17(6):2084-92.

25. Bilardi J, Walker S, Read T, et al. Gay and bisexual men's views on rapid self-testing for HIV. AIDS Behav. 2013;17(6):2093-9.

26. Greacen T, Friboulet D, Blanchier A, et al. Internet-using men who have sex with men would be interested in accessing authorised HIV self-tests available for purchase online. AIDS Care. 2013;25(1):49-54.

27. Wei C, Yan H, Yang C, et al. Accessing HIV testing and treatment among men who have sex with men in China: a qualitative study. AIDS Care. 2013;26(3):372-8.

28. Wong V, Johnson C, Cowan E, et al. HIV self-testing in resourcelimited settings: regulatory and policy considerations. AIDS Behav. 2014. doi:10.1007/s10461-014-0825-9.
29. Nersesian P, Hülsmann M, Cloutier S, Chintalova-Dallas R. Assessment of over-the-counter HIV rapid test kits in Namibia. Arlington: USAID's AIDS Support and Technical Assistance Resources, AIDSTAR-One, Task Order 1; 2013 [cited 2014 March 17]. http://www.aidstar-one.com/sites/default/files/AID STAR-One_Report_Namibia_OTC_Rapid_Test_Kits.pdf.

30. Southern African AIDS Trust and Wits Reproductive Health and HIV Institute. Consultative meeting on HIV self-testing;Gauteng, South Africa; 25-26 Mar 2014.

31. Peck R, MuChen Lin J, van Rooyen H, et al. What should the ideal HIV self-test look like? A usability study of test prototypes in unsupervised HIV self-testing in Kenya, Malawi, and South Africa. AIDS Behav. 2014. doi:10.1007/s10461-014-0818-8.

32. Dong M, Regina R, Hlongwane S, Ghebremichael M, Wilson D, Dong K. Can laypersons in high-prevalence South Africa perform an HIV self-test accurately? [Abstract A-641-0087-10374]. In:Presented at 20th International AIDS Conference; Melbourne, Australia; 20-25 July 2014.

33. Scott A. Self-testing as part public health screening for HIV in resource-poor environments: some ethical considerations. AIDS Behav. 2014. doi:10.1007/s10461-014-0833-9.

34. Allais L, Venter F. The ethical, legal and human rights concerns raised by licensing HIV self-testing for private use. AIDS Behav. 2014. doi:10.1007/s10461-014-0823-y.

35. Brown A, Djiemu E, Cameron D. Is there evidence of harms from self-tests? A rapid evidence search. AIDS Behav. 2014. doi:10. 1007/s10461-014-0831-y.

36. Billy S. Personal communication, Feb 282014.

37. Cambiano V, Napierala Mavedezenge S, Phillips A, et al. Modelling the potential population impact and cost-effectiveness of self-testing for HIV: evaluation of data requirements. AIDS Behav. 2014. doi:10.1007/s10461-014-0824-x.

38. Ventuneac A, Carballo-Dieguez A, Leu C, et al. Use of a rapid HIV home test to screen sexual partners: an evaluation of its possible use and relative risk. AIDS Behav. 2009;13(4):731-7.

39. Katz D, Golden D, Stekler J. Replacing clinic-based tests with home-use tests may increase HIV prevalence among Seattle men who have sex with men: evidence from a mathematical model. Sex Transm Dis. 2014;41(1):2-9.

40. Shank L, Klarkowski D, O'Brien DP. False positive HIV diagnoses in resource limited settings: operational lessons learned for HIV programmes. PLoS Med. 2013;8(3):e59906. 